\title{
Weighted Non-locally Self-similarity Sparse Representation for Face Deblurring
}

\author{
Lei $\operatorname{Tian}^{1}$, Chunxiao Fan ${ }^{1}$, Yue Ming ${ }^{1}$, Xiaopeng Hong ${ }^{2}$ \\ ${ }^{1}$ Beijing Key Laboratory of Work Safety Intelligent Monitoring, School of Electronic \\ Engineering, Beijing University of Posts and Telecommunications, Beijing, P.R.China. \\ ${ }^{2}$ Department of Computer Science and Engineering, University of Oulu, Finland.
}

\begin{abstract}
The human face is one of the most interesting subjects in various computer vision tasks. In recent years, significant progress has been made for generic image deblurring problem, but existing popular sparse representation based deblurring methods are not able to achieve excellent results on blurry face images. The failure of these methods mainly stems from the lack of local/non-local self-similarity prior knowledge. There are many similar non-local patches in the neighborhood of a given patch in a face image, therefore, this property should be effectively exploited to obtain a good estimation of the sparse coding coefficients. In this paper, we introduce the current weighted nonlocally self-similarity (WNLSS) method [1], which is originally proposed to remove the noise for natural images, into the face deblurring model. There are two terms in the WNLSS sparse representation model, data fidelity term and regularization term. Based on the theoretical analysis, we show the properties of data fidelity term and regularization term also can fit well for face deblurring problem. The results also demonstrate that WNLSS method can achieve excellent performance in terms of both synthetic and real blurred face dataset.
\end{abstract}

\section{Introduction}

Restoring a clear image from one blurred image has many important applications, such as video surveillance, remote sensing and so on. The process under a spatially-invariant model can be generally formulated as

$$
B=k * I+\varepsilon,
$$

where $I$ is the latent sharp image, $B$ is the blurred (observed) image, $k$ and $\varepsilon$ denote the blurred kernel and the noise term, respectively. In the past decades, extensive studies have been conducted on the natural image deblurring problem [2-8]. Since the ill-posed nature of image deblurring, additional prior knowledge is need to constrain the solutions of Eq (1), such as heavy-tailed gradient distributions $[3,9]$, regularization-based techniques $[6,10]$ and sparsity constraints $[11,12]$. Though the above prior knowledge and models work well for natural images, they can not perform well when faced with specific object category, such as face image. Compared with natural image, the face image has 
special non-local self-similarity (NSS) and less texture information, the work [13] exploits the NSS patches to reconstruct the visual light face images from the near infrared images. Since face is one of the most interesting objects in various computer vision application, we focus on face image deblurring in this paper.

The success of state-of-the-art image deblurring methods $[2,14,15]$ mainly depends on extraction of salient edges for kernel estimation. However, the edge prediction step usually does not work well when faced with those blurred images with less texture, such as face image, which is demonstrated in the work [8]. As an alternative, the sparsity representation based deblurring methods have achieved excellent results for natural image deblurring. The sparse representation of a clear image $I$ and an observed image $B$ can be denoted as following:

$$
\min _{a_{I}}\left\{\left\|I-D a_{I}\right\|+\lambda\left\|a_{I}\right\|_{p}\right\}
$$

and

$$
\min _{a_{B}}\left\{\left\|B-k * D a_{B}\right\|+\lambda\left\|a_{B}\right\|_{p}\right\}
$$

where $\|\bullet\|_{p}$ is $p$-norm term ( $p$ is set to be 0 or 1 ) and it is expected that the coding coefficients $a_{I}$ and $a_{B}$ are sparse as much as possible. $D$ denotes a learningbased over-completed dictionary and the parameter $\lambda$ controls the contribution of regularization item. It is impossible for us to obtain the $I$ and its coding coefficients $a_{I}$, so we hope the coding coefficients of observed image $a_{B}$ which is learned from our deblurring model could be close enough to $a_{I}$. Therefore, the reconstructed version of original image $\hat{I}=D a_{B}$ is considered as the final deblurrring result. Due to $l_{0}$-minimization problem is a NP-hard problem, the $l_{1}$-minimization problem is usually used in sparse representation model.

\subsection{Motivation}

For a blurred face image, it is very challenging to solve the sparse codes $a_{B}$ of observed image $B$ just by using sparsity constraint prior, just like Eq (3). The universal prior knowledge easily leads to the solution of Eq (3) entrap into a local minimum and we need more specific prior knowledge to design the objective function of facial deblurring model. In this paper, we apply the existing WNLSS sparse representation model [1] for the face image deblurring task. It is well known that there are lots of non-local similar patches in a face image, as shown in Figure 1. The self-similarity based coding coefficients $a_{z}$ can be computed, so that the more accurate estimation of sparse coding coefficients $a_{B}$ can be obtained by exploiting these non-local redundancies. Moreover, many existing sparse representation based deblurring methods $[16,17]$ take observed image $B$ as a long vector and obtain a global coding coefficients for the whole image by solving the Eq (3). However, the solution of this kind of deblurring model has been proved is unstable and time-consuming [18]. In order to make the computational process stable and fast, we should provide smaller input signal for deblurring model. Due to the face image has the NSS property, a given 
local patch can be reconstructed well by referencing its similar patches. We intend to extract the dense local patches from the observed image and fed them into our sparse representation model. So the input size of sparse representation model is relatively small and we can obtain the stable and effective coding coefficients for each local patch. To further reduce the patch-wise residual, the weighted encoding technique [1] is introduced into the data fidelity term of WNLSS method. The large pixel residual will lead to the reconstructed local patch produce the ghost or ringing visual artifacts. The pixel $i$ which has large pixel residual is expected to assign small weights, so that this pixel's effect on the encoding of observed image over over-completed dictionary can be reduced.

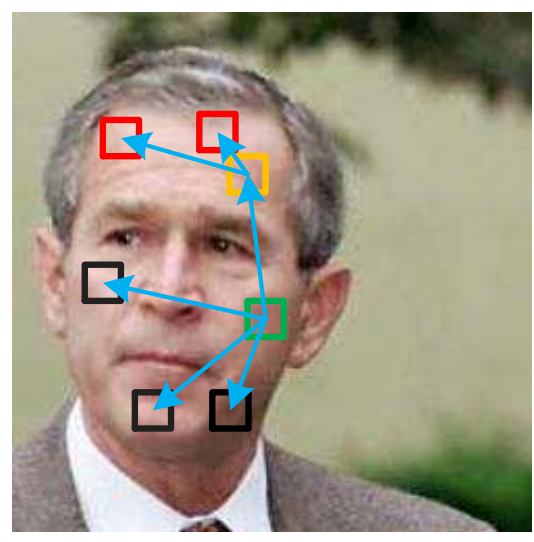

Fig. 1. The illustration of non-local self-similarity in a face image. This property brings us an alternative approach to estimate the sparse coding coefficients of reconstruction signal. For solving the real coding coefficients of a given blurred patch, we just need to search a set of similar patches and reconstruct this patch by linearly combining these similar patches. Then, we estimate the real coding coefficients of a given blurred patch by using the coding coefficients of the reconstructed patch.

The rest of the paper is organized as follows. we describe the deblurring method in the Section 2 and investigate the performance of our method on both synthetic and real blurry face datasets in Section 3 and conclude this paper in Section 4 .

\section{Weighted non-locally self-similarity (WNLSS) sparse representation}

For an image $I \in \mathbb{R}^{M \times N}$ and $I_{i}$ denotes a local patch extracted at pixel $i$ with size $m \times n$. The $i$-th lcoal patch $I_{i}$ of $I$ can be sparsely encoded as $I_{i}=D a_{I, i}$. In order to deblur $I$ from $B, B$ need to be sparsely coded with the learned dictionary $D$ by solving the $\mathrm{Eq}(3)$. Table 1 summary some notations which are frequently used in our paper. 
Table 1. The notation table

\begin{tabular}{c|l}
\hline Notations & Descriptions \\
\hline $\mathbf{I}$ & The latent sharp (clear) image. \\
$\mathbf{B}$ & The observed (blurred) image. \\
$\mathbf{D}$ & The dictionary of sparse representation model. \\
$\mathbf{a}_{\mathbf{I}}$ & The sparse coding coefficients of clear image $I$. \\
$\mathbf{a}_{\mathbf{B}}$ & The sparse coding coefficients of observed image $B$. \\
$\mathbf{a}_{\mathbf{z}}$ & The estimated sparse coding coefficients based on facial NSS redundancies. \\
$\mathbf{k}$ & The blur kernel (known in this paper). \\
$\mathbf{e}$ & The residual vector between original signal and reconstructed signal. \\
$\mathbf{W}$ & The weight matrix of weighted coding based data fidelity term. \\
\hline
\end{tabular}

\subsection{The Dictionary Learning}

In this section, we first discuss the dictionary $D$ learning problem. Many existing deblurring methods need some example images to learn dictionary $[6,8]$ and this kind of example images are required to choose elaborately, so that the learned dictionary can contain indispensable texture units. Therefore, the usage of this kind of deblurring methods is restricted. Compared with this kind of methods, we intend to learn dictionary $D$ from the input image itself. If we learn an universal dictionaries from the whole original image by using K-SVD [19], the such over-completed dictionary may leads to expensive computational cost and unstable solutions, this observation has been proved by the work [12]. How to obtain the stable and properly sparse solution is a key problem in the dictionary learning process. Inspired by the work [20], all patches of the input image are clustered into $K$ centers by the traditional K-Means algorithm. Other complicated clustering algorithms are also investigated in our experiment, such as Gaussian Mixture Model, mean-shift and spectral clustering, but there is no significant improvement of deblurring performance. So we select the KMeans algorithm due to its simplicity. The problem of dictionary selection can be denoted as follow:

$$
\min _{D_{k}, C_{k}}\left\{\left\|X_{k}-D_{k} C_{k}\right\|_{2}^{2}+\lambda\left\|C_{k}\right\|_{1}\right\}
$$

where $X_{k}$ denotes the $k$ th clustered set and $C_{k}$ is the coding coefficient matrix over cluster-based dictionary $D_{k}$. Because of the elements in each cluster $X_{k}$ is similar, it is not necessary to form an over-completed dictionary in order to describe the variation of $X_{k}$. So the compact representation is better than overcompleted representation due to the computational cost of dictionary learning. For each $X_{k}$, we compute the principal components by PCA algorithm. To compactly represent the discriminative pattern of each cluster set $X_{k}$, we just select $L$ eigenvectors $\left[p_{1}, \cdots, p_{l}, \cdots, p_{L}\right]$ corresponding to the largest eigenvalues to construct the dictionary $D_{k}$.

For each patch need to be coded, only one sub-dictionary is adaptively selected to code it and the coding coefficients of other all sub-dictionaries is set 
to be 0 . Therefore, the sparse constraint in the existing sparsity-based deblurring is also satisfied.

\subsection{Formulation}

In order to make the Eq (3) can work well on face image deblurring problem, the weighted coding technique [1] and facial non-local self-similarity prior knowledge is introduced into the data fidelity term $\|(B-k * D a)\|_{2}^{2}$ and regularization term $R(a)$ of the WNLSS method, respectively. We will particularly describe these two terms in the following parts.

Weighted coding based data fidelity term It is well known that the residual between the deblurred image and observed image is directly related to the performance of reconstruction method. It is naturally expected that the effect of those pixels with large residual can be decreased. In order to minimize the data fitting residual $\|B-k * D a\|_{2}^{2}$ for all pixels, the weighted coding technique is employed to weight the residual. Assume the overall residual vector is defined as following:

$$
e=\left[e_{1}, e_{2}, \cdots, e_{N}\right]=B-k * D a,
$$

where $e_{i}=B_{i}-k * D a_{i}$ and $e_{1}, e_{2}, \cdots, e_{N}$ are i.i.d samples. According to modern robust estimation technique, each residual should be assigned a proper weight, like $\bar{e}_{i}=w_{i}^{1 / 2} e_{i}$. Therefore, Eq (3) can be re-written as following:

$$
\min _{W, a_{B}}\left\{\left\|W^{1 / 2}\left(B-k * D a_{B}\right)\right\|_{2}^{2}+\lambda\left\|a_{B}\right\|_{p}\right\},
$$

where $W$ is a diagonal weight matrix with $W_{i i}=w_{i}$. Obviously, the weight $w_{i}$ and the magnitude of residual $e_{i}^{2}$ should have inverse property, so the relationship between these two items can be simply formulated as following:

$$
w_{i}=\exp \left(-c_{0} e_{i}^{2}\right)
$$

where $c_{0}$ is the penalization parameters of the residual $e_{i}$.

Non-local self-similarity based regularization term As above mentioned, we expect $a_{B}$ is close to $a_{I}$ as much as possible, but $a_{I}$ is unknown for face deblurring task. Fortunately, there is a great number of non-local redundancies in face images, regardless of clear images or blurred images. So it can be expected that providing a good estimation $a_{z}$ for $a_{I}$ by exploiting the self-similarity redundancies prior knowledge.

For a given patch $I_{i}$, a set of similar non-local patches $I_{i}^{p}$ are collected in a large window around $I_{i}$. We select $P$ most similar patches $I_{i}^{p}$ to $I_{i}$ and assign them different weight to predict the $\hat{I}_{i}$ as $\hat{I}_{i}=\sum_{p=1}^{P} d_{i}^{p} I_{i}^{p}$, where $P$ denotes the 
number of similar patches, and the weight $d_{i}^{p}$ can be computed as a traditional regularized least-square problem:

$$
\min _{d_{i}}\left\|I_{i}-I d_{i}\right\|_{2}^{2}+\eta\left\|d_{i}\right\|_{2}^{2},
$$

where $I=\left[I_{i}^{1}, I_{i}^{2}, \cdots, I_{i}^{P}\right]$ and $d_{i}=\left[d_{i}^{1}, d_{i}^{2}, \cdots, d_{i}^{P}\right]^{T}$. The regularization term $\left\|d_{i}\right\|_{2}^{2}$ is applied to enhance the stability of least-square solution and $\eta$ is the regularization parameter. Therefore, each patch $I_{i}$ can be reconstructed by a set of non-local similar patches $I_{i}^{p}$. In other words, for an observed images, each patch $I_{i}$ and its NSS based reconstruction $\hat{I}_{i}$ can be denoted as $I_{i}=D_{i} a_{B, i}$ and $\hat{I}_{i}=D_{i} a_{z, i}$. Accordingly, the $a_{z, i}$ for the patch $\hat{I}_{i}$ can be obtained as:

$$
a_{z, i}=D_{i}^{T} \hat{I}_{i}
$$

In order to make the reconstruction $\hat{I}_{i}$ is close enough to $I_{i}$, obviously, the coding coefficients of non-local reconstruction $a_{z, i}$ should be similar as that of observation $a_{B, i}$. Therefore, the NSS information is introduced into the objective function and the $\mathrm{Eq}(6)$ can be formulated as following:

$$
\min _{W, a_{B}}\left\{\left\|W^{1 / 2}\left(B-k * D a_{B}\right)\right\|_{2}^{2}+\lambda\left\|a_{B}-a_{z}\right\|_{1}\right\} .
$$

It is the final objective function in our method, and the optimization process is detailed in the next section.

\subsection{Optimization}

Assumed the blurring kernel $k$ and dictionary $D$ are given, there are two variables $W$ and $a_{B}$ in the proposed formulation, we can effectively solve them by updating one variable and fixing another.

Updating $\boldsymbol{a}_{B}$ and fixing $\boldsymbol{W}$ When $W$ is fixed, the objective function of our method becomes the $\ell_{1}$-norm sparse coding problem. This problem can be effectively solved via the iterative re-weighted scheme [21]. According to the deduction of work [21], the $(k+1)$ th sparse coding $a_{B}^{(k+1)}$ can be denoted as:

$$
a_{B}^{(k+1)}=\left(D^{T} W D+V^{(k+1)}\right)^{-1}\left(D^{T} W B-D^{T} W D a_{z}\right)+a_{z},
$$

where $V$ is a diagonal matrix and it can be initialized as an identity matrix. And the diagonal element $V_{i i}^{(k+1)}$ of auxiliary matrix $V^{(k+1)}$ can be updated as:

$$
V_{i i}^{(k)}=\lambda /\left(\left(a_{B, i}^{(k)}-a_{z, i}^{(k)}\right)^{2}+\varepsilon^{2}\right)^{1 / 2}
$$

where $\varepsilon$ is used to avoid the singular problem of solving process. The $a_{B}^{(k)}$ and $a_{z}^{(k)}$ are the $i$-th element in the $k$-th iteration. 
Updating $\boldsymbol{W}$ and fixing $\boldsymbol{a}_{B}$ Due to we have updated the observed coding coefficients $a_{B}$ and NSS based coding coefficients $a_{z}$ in the above section, therefore, the residual $e^{(k)}$ can be computed as $e^{(k)}=B-\hat{I}^{(k)}$. Further, the weight elements $w_{i i}^{(k)}$ in the $k$-iterations can be updated by the Eq (7).

Algorithm 1 summarizes the whole procedure of our deblurring method.

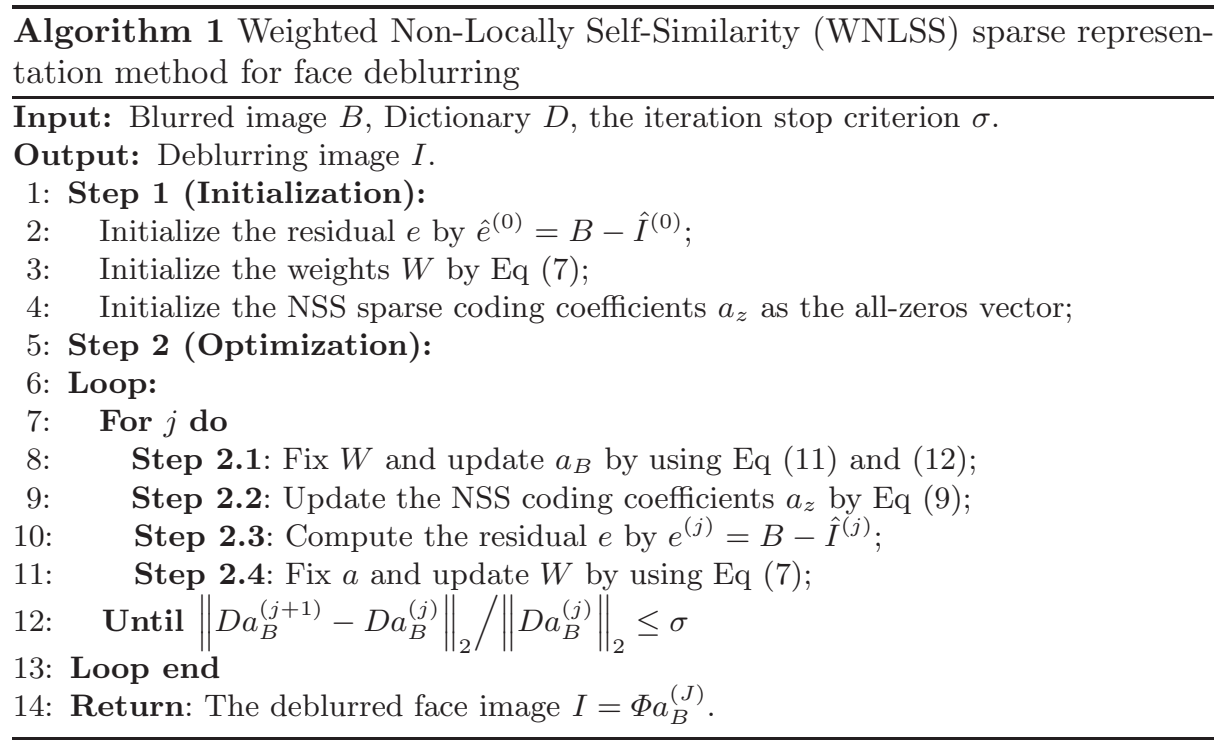

\section{$3 \quad$ Experiments}

In this section, we first investigate the performance of the WNLSS model on both synthetic and real blurred face images in Section 3.1 and 3.2, respectively. To evaluate the quality of deblurring face image, we compute the Peak Signalto-Noise Ratio (PSNR) and perceptual quality metric FSIM [22] in this section. The basic parameters of WNLSS are set to be as follows: The size of local patch $[m, n]$ is $[7,7]$, the dictionary size $K$ is 200 , the number of eigenvectors $L$ is 70 , the regularization parameter $\lambda$ is 0.1 and the iteration number $J$ is 10 .

\subsection{Evaluate on synthetic blur images}

For synthetic blurred image, we first use two widely applied blur kernels (i.e., uniform kernel and Gaussian kernel) to convolute the clear face images. Then the synthetic blurred images with $r \times r$ uniform blur kernel and 2D Gaussian blur kernel with standard deviation $s$ can be obtained. In order to demonstrate the robustness of the WNLSS method when faced with noise, we add some additive white Gaussian noise (AWGN) into the blurred images. As the section 
of related work mentioned, we mainly compare the three kinds of deblurring method, i.e., (1) the universal sparse representation based deblurring methods $[15,14,23,24] ;(2)$ face image based deblurring methods [8] and (3) natural image based deblurring methods $[3,2,4,25]$.

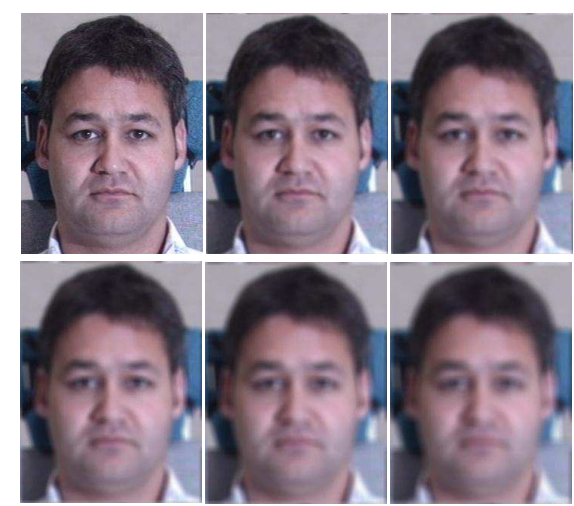

(a) The Uniform Blur Kernel

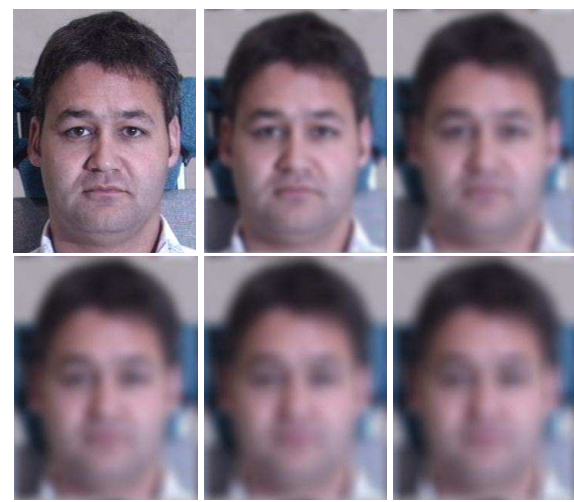

(b) The Gaussian Blur Kernel

Fig. 2. Examples of synthetic blurred face images with different blur parameters. (a) The blurred face image with uniform kernel. From left to right and top to bottom is that: original face image, the size of uniform kernel is $5 \times 5,7 \times 7,9 \times 9,11 \times 11,13 \times 13$. (b) The blurred face image with Gaussian kernel. From left to right and top to bottom is that: original face image, blurred face image with standard deviation 3, 5, 7, 9, 11.

Results on The Uniform Kernel We vary the size of uniform kernel $r \times r$ from 5 to 13 with step size 2 . The blurred samples with uniform kernel are listed in Figure 2(a). The value of PSNR and FSIM on these blurred images of different deblurring methods are reported in Table 2. The deblurring results of $9 \times 9$ uniform kernel are also listed in Figure 3 .

We can observe that the WNLSS method and the work [23] can achieve large PSNR and FSIM improvement compared with other deblurring methods from Table 2 and the uniform blur is basically removed in the face image from Figure 3. The WNLSS method achieves better performance than other all methods on all uniform blur cases. Though the work [8] is specially designed for face deblurring problem, it requires a large enough exemplars dataset for the match of face exemplar structure and its performance directly depends on the initialization of predicted salient edges. When the exemplar dataset is not large enough, the poor predicted edges may lead to the failure of kernel estimation from exemplar structure. The other works also introduce the prior knowledge of sparsity and edge selection into their models, but they do not achieve excellent performances as our method. On the one hand, they do not consider the special structural priors of face image and ignore the non-local self-similarity information. On the other hand, compared with other sparse based deblurring methods, the weighted 
coding technique is introduced to further optimize the overall residual of a face image. Therefore, the WNLSS achieve better deblurring performance on face image.

Table 2. The values of PSNR (FSIM) by different methods with different size of uniform kernel

\begin{tabular}{|c|c|c|c|c|c|c|c|c|}
\hline Kernel Size & & 5 & & 7 & 9 & & 11 & 13 \\
\hline Jia [15] & $\mid 37.61$ & $(0.9100)$ & 35.30 & $(0.8438)$ & $34.68(0.8244)$ & 33.61 & $(0.7524)$ & $33.05(0.7517)$ \\
\hline Krishnan [14] & 37.11 & $(0.8947)$ & 35.96 & $(0.8540)$ & $34.66(0.8044)$ & 33.85 & 605) & $32.97(0$. \\
\hline Portilla [24] & 36.53 & $(0.8737)$ & 34.70 & $(0.8004)$ & $33.62(0.7373)$ & 32.82 & $(0.6838)$ & $32.17(0.6365)$ \\
\hline Kheradmand [23] & 36.96 & $(0.8152)$ & 36.67 & $(0.8096)$ & $36.17(0.8187)$ & 36.13 & $(0.8267)$ & $36.13(0.8325)$ \\
\hline Pan $[8]$ & 39.55 & $(0.9167)$ & 37.50 & $(0.8845)$ & $35.56(0.8350)$ & 34.51 & $(0.8017)$ & $34.34(0.7997)$ \\
\hline Shan [3] & 36.88 & $(0.8642)$ & 35.60 & $(0.8291)$ & $34.74(0.8012)$ & 33.34 & $(0.7202)$ & $32.63(0.6651)$ \\
\hline Cho [2] & 36.81 & $(0.8504)$ & 34.63 & $(0.8032)$ & $33.83(0.7410)$ & 33.56 & $(0.7017)$ & $33.04(0.6653)$ \\
\hline $\mathrm{Xu}[4]$ & 37.99 & $(0.9137)$ & 35.86 & $(0.8651)$ & $35.48(0.8479)$ & 34.77 & $(0.8336)$ & $34.06(0.8051)$ \\
\hline Beck [25] & 37.16 & $(0.8210)$ & 36.84 & $(0.8285)$ & $36.12(0.8234)$ & 35.50 & $(0.8141)$ & $34.90(0.8058)$ \\
\hline Ours & 39.86 & $(0.8966)$ & 38.78 & $(0.8836)$ & $37.86(0.8703)$ & 37.29 & $(0.8617)$ & $36.61(0.8500)$ \\
\hline
\end{tabular}

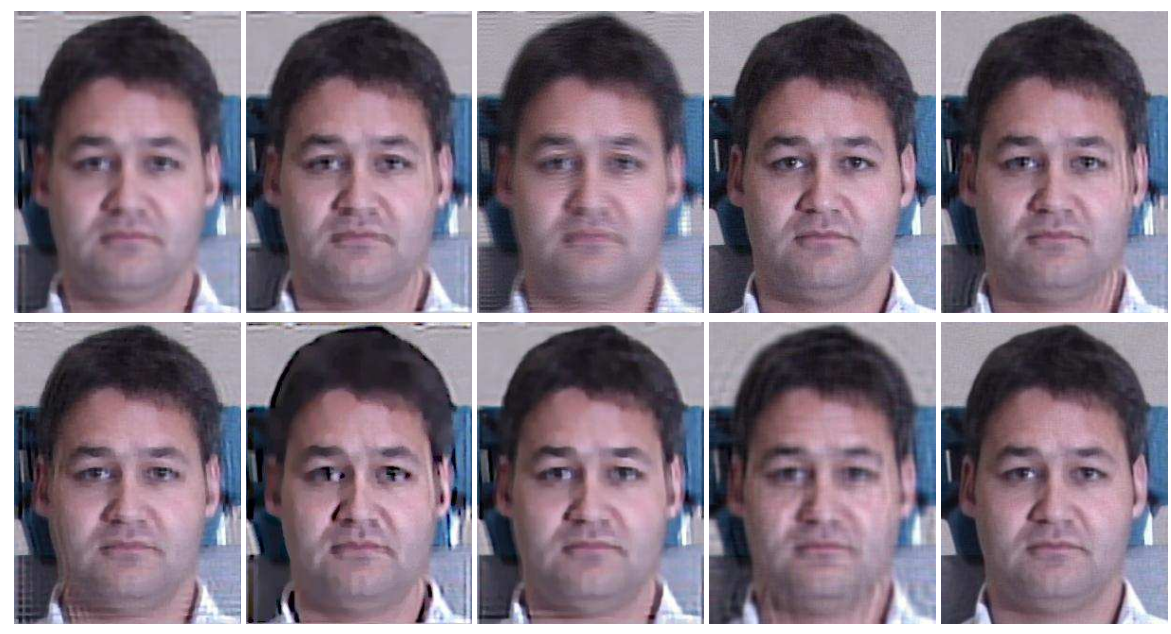

Fig. 3. The comparison of deblurring performance on the face image with $9 \times 9$ uniform kernel. From left to right and top to bottom is that: [15], [14], [24], [23], [8], [3], [2], [4], [25] and our WNLSS.

Results on The Gaussian Kernel In this subsection, we set the size of Gaussian kernel to be $25 \times 25$ and the standard deviation from 3 to 11 with step size 2. The blurred samples with Gaussian kernel are listed in Figure 2(b). The value of PSNR and FSIM on these blurred images of different deblurring 
methods are reported in Table 3. The deblurring results of Gaussian kernel with standard deviation 3 are also listed in Figure 4.

Different from the observation of uniform kernel, our method work better than other methods on small Gaussian blur and the work [23] can achieve better performance on large blur cases. For a noisy and blurred image, the work [23] first need to remove the contribution of the noise and then perform the deblurring operation. And the performance of denoising is directly related to the initialization of blurring matrix. However, our method need not to explicitly remove the AWGN of the face image and the objective function of WNLSS is naturally robust to the AWGN. What is more, the computational cost in terms of time complexity of the work [23] can also not be ignored. Compared with the single loop in the WNLSS model, the work [23] has the outer loop and inner loop. Therefore, it takes more computational time for large blur, regardless of encountering the uniform kernel or Gaussian kernel. For the Gaussian blur kernel with standard deviation 5, the work [23] takes 26.94 seconds and our method only takes 13.08 seconds in a PC with $2.0 \mathrm{GHz}$ CPU, the WNLSS method just use half computational time to achieves similar performance with the work [23]. Compared with other deblurring methods except the work [23], the WNLSS method also achieves better PSNR and FSIM performance due to the introduction of weighted coding and non-local self-similarity priors.

Table 3. The values of PSNR (FSIM) on the Gaussian kernel with different standard deviation of face image

\begin{tabular}{l|c|c|c|c|c}
\hline Standard Deviation & 3 & 5 & 7 & 9 & 11 \\
\hline Xu and Jia [15] & $34.69(0.8381)$ & $32.21(0.7256)$ & $31.79(0.6965)$ & $31.45(0.6708)$ & $31.20(0.6513)$ \\
Krishnan [14] & $35.44(0.8452)$ & $33.63(0.7692)$ & $32.46(0.7179)$ & $31.65(0.6787)$ & $31.28(0.6511)$ \\
Portilla [24] & $33.57(0.7523)$ & $31.95(0.6787)$ & $31.27(0.6430)$ & $30.97(0.6262)$ & $30.82(0.6163)$ \\
Kheradmand [23] & $37.16(0.8804)$ & $\mathbf{3 5 . 1 0}(0.8230)$ & $\mathbf{3 4 . 0 4}(0.7908)$ & $\mathbf{3 4 . 2 5}(0.8030)$ & $\mathbf{3 4 . 3 6}(0.8081)$ \\
\hline Pan [8] & $36.48(0.8711)$ & $34.29(0.7995)$ & $32.80(0.7401)$ & $32.34(0.7298)$ & $32.06(0.7215)$ \\
\hline Shan [3] & $34.97(0.8271)$ & $33.44(0.7643)$ & $32.09(0.6991)$ & $31.47(0.6516)$ & $31.30(0.6216)$ \\
Cho [2] & $34.97(0.7805)$ & $32.35(0.6611)$ & $30.10(0.6426)$ & $31.08(0.6145)$ & $31.07(0.6207)$ \\
Xu [4] & $34.06(0.8126)$ & $33.32(0.7557)$ & $32.47(0.7265)$ & $32.12(0.7181)$ & $31.91(0.7151)$ \\
Beck [25] & $36.28(0.8314)$ & $34.51(0.7884)$ & $33.18(0.7392)$ & $32.91(0.7309)$ & $32.76(0.7219)$ \\
\hline Ours & $\mathbf{3 7 . 2 9}(0.8674)$ & $34.75(0.7983)$ & $33.44(0.7561)$ & $33.59(0.7693)$ & $33.51(0.7725)$ \\
\hline
\end{tabular}

\subsection{Evaluate on real blur images}

For real blurred image, we select two real blurred image from the Point-andShoot Cameras (PaSC) [26]. The face images from PaSC dataset are shot by the point-and-shoot camera, therefore, there are not only motion blur but also poor focus. Since the focus of our work is to restore the blurred image by the known kernel, we borrow the motion blur kernel estimation from the work [27] to estimate the blur kernel. What is more, it it impossible for us to obtain the original clear face image from the PaSC dataset, therefore, we can not compute the PSNR and FSIM values like the above section. We 


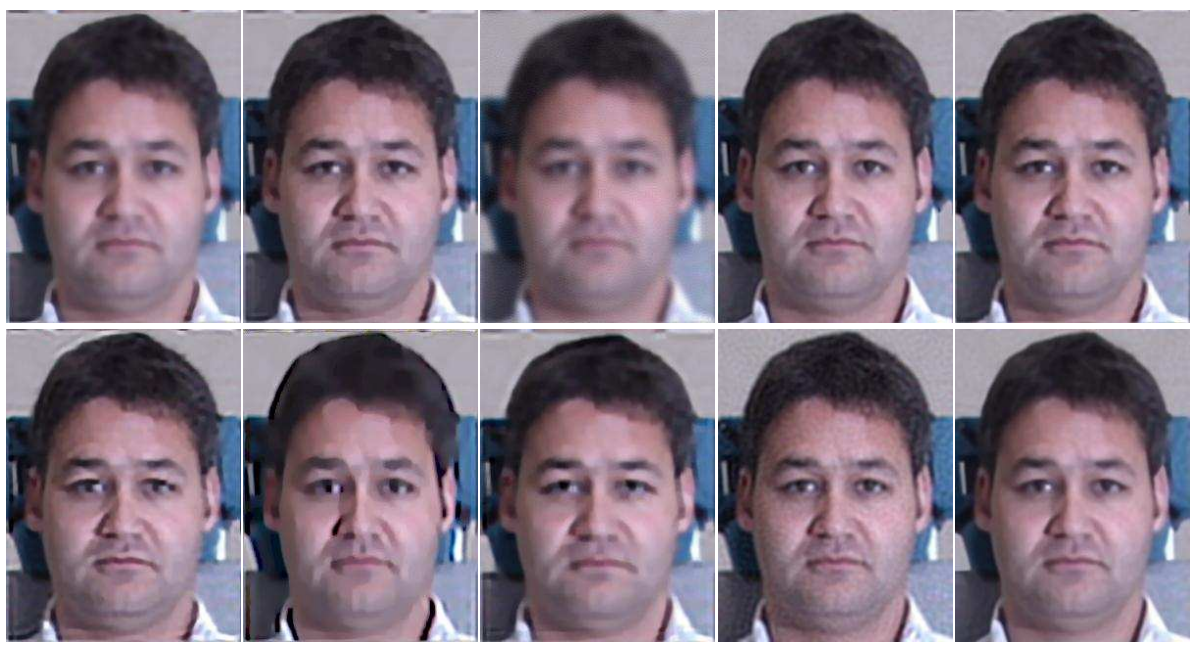

Fig. 4. The comparison of deblurring performance on the face image with Gaussian kernel of standard deviation 5. From left to right and top to bottom is that: [15], [14], [24], [23], [8], [3], [2], [4], [25] and our WNLSS.

just evaluate the deblurring performance by visual observation. The deblurring performance of the work [8], the work [23] and our work is significantly better than other methods. Therefore, for a more difficult deblurring task, we just compare these methods on real blur face images.

We show the original blurred face image and deblurring results by the work [8], the work [23] and our work in Figure 5 and Figure 6. We can observe that the face image are deblurred by the WNLSS method are much clear than other methods. Since the WNLSS method can accurately restructure a local patch by referencing other similar patches and further reduce the coding residual by using the weighted coding methodology, therefore, it can restructure more detail information on real blurred and noisy face images. The estimated kernels from [27] have some bias with the real unknown blur kernels, but it still achieves excellent performances in this case, it further demonstrates the WNLSS method is robust to the kernel estimation error. Though the work [8] is specially designed for face deblurring problem, it requires a large enough exemplars dataset for the match of face exemplar structure and its performance directly depends on the initialization of predicted salient edges. For the blurred Figure 5, we manually locate the initial contours of blurred face images and fed the contours information into their model, and for the blurred Figure 6, we search its best matched contours in the author provided exemplar dataset. The work [8] produces some visual ringing and ghost artifacts in the Figure 6, and the deblurring result are much worse than other two methods when there is a mismatch between the predicted salient edges and real edges (e.g. Figure 6). For another work [23], since it is designed by using normalized graph Laplacian, it works well when 
faced with the structural blur kernel (i.e., Gaussian kernel). However, it can not restore more facial detail information by using the pre-defined structural prior knowledge when faced with real blur image. Moreover, this work [23] comprises of outer and inner loop and takes a large amount of time to deblur the complicated blurred image.

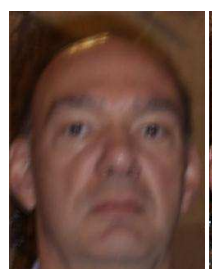

(a)

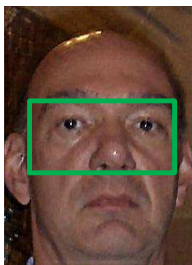

(b)

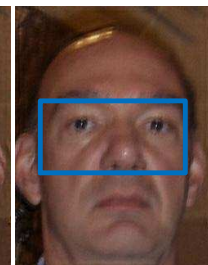

(c)

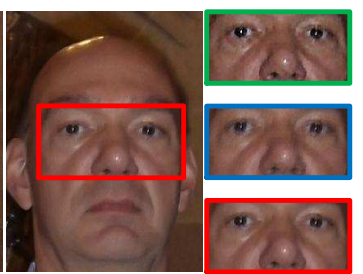

(d)

(e)

Fig. 5. The deblurring performance comparison on real blurred face images. From left to right is that: original real image, deblurred image by [8], deblurred image by [23], deblurred image by our WNLSS and close-up view for detail presentation.

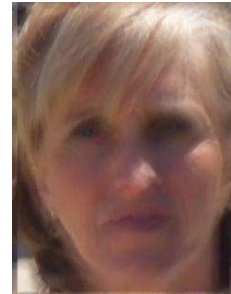

(a)

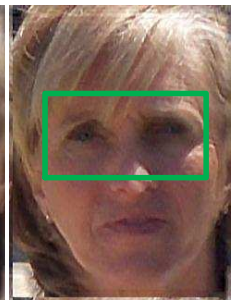

(b)

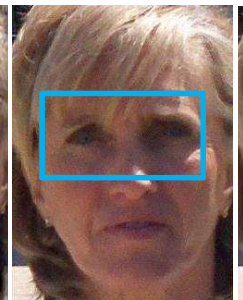

(c)

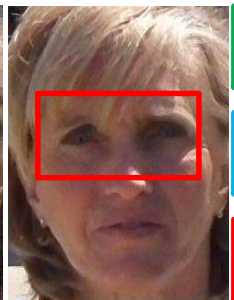

(d)

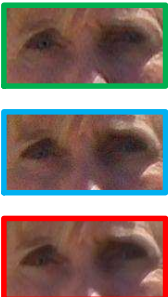

(e)

Fig. 6. The deblurring performance comparison on real blurred face images. From left to right is that: original real image, deblurred image by [8], deblurred image by [23], deblurred image by our WNLSS and close-up view for detail presentation.

\section{Conclusion}

In this paper, we introduce the existing weighted non-local self-similarity sparse model [1] into face deblurring task and verify that the model is suitable for face image deblurring. In theory, on the one hand, the weighted coding technology can effectively reduce the coding residual of sparse representation model, so that the pixels with large residual have smaller effect on the whole deblurring process. On the other hand, the estimation of real unknown coding coefficients can be learned by exploiting the NSS property of face image. Extensive results on synthetic and real blurred face image show the WNLSS achieves competitive 
performance compared with other state-of-the-art methods and demonstrates the effectiveness and robustness of the WNLSS method.

Acknowledgement. The work presented in this paper was supported by the National Natural Science Foundation of China (Grants No. NSFC-61402046), Fund for Beijing University of Posts and Telecommunications (No.2013XZ10, 2013XD-04), Fund for the Doctoral Program of Higher Education of China (Grants No.20120005110002).

\section{References}

1. Jiang, J., Zhang, L., Yang, J.: Mixed noise removal by weighted encoding with sparse nonlocal regularization. IEEE transactions on image processing 23 (2014) 2651-2662

2. Cho, S., Lee, S.: Fast motion deblurring. In: ACM SIGGRAPH Asia 2009 Papers. SIGGRAPH Asia '09, New York, NY, USA, ACM (2009) 145:1-145:8

3. Shan, Q., Jia, J., Agarwala, A.: High-quality motion deblurring from a single image. ACM Trans. Graph. 27 (2008) 73:1-73:10

4. Xu, L., Jia, J.: Two-phase kernel estimation for robust motion deblurring. In: European conference on computer vision, Springer (2010) 157-170

5. Mairal, J., Bach, F., Ponce, J., Sapiro, G., Zisserman, A.: Non-local sparse models for image restoration. In: 2009 IEEE 12th International Conference on Computer Vision, IEEE (2009) 2272-2279

6. Dong, W., Zhang, L., Shi, G., Wu, X.: Image deblurring and super-resolution by adaptive sparse domain selection and adaptive regularization. IEEE Transactions on Image Processing 20 (2011) 1838-1857

7. Sun, L., Cho, S., Wang, J., Hays, J.: Edge-based blur kernel estimation using patch priors. In: Computational Photography (ICCP), 2013 IEEE International Conference on, IEEE (2013) 1-8

8. Pan, J., Hu, Z., Su, Z., Yang, M.H.: Deblurring face images with exemplars. In: European Conference on Computer Vision, Springer (2014) 47-62

9. Levin, A., Weiss, Y., Durand, F., Freeman, W.T.: Efficient marginal likelihood optimization in blind deconvolution. In: Computer Vision and Pattern Recognition (CVPR), 2011 IEEE Conference on, IEEE (2011) 2657-2664

10. Oliveira, J.P., Bioucas-Dias, J.M., Figueiredo, M.A.: Adaptive total variation image deblurring: a majorization-minimization approach. Signal Processing $\mathbf{8 9}$ (2009) 1683-1693

11. Cai, J.F., Ji, H., Liu, C., Shen, Z.: Framelet-based blind motion deblurring from a single image. IEEE Transactions on Image Processing 21 (2012) 562-572

12. Dong, W., Shi, G., Hu, X., Ma, Y.: Nonlocal sparse and low-rank regularization for optical flow estimation. IEEE Transactions on Image Processing 23 (2014) $4527-4538$

13. Chen, J., Yi, D., Yang, J., Zhao, G., Li, S.Z., Pietikainen, M.: Learning mappings for face synthesis from near infrared to visual light images. In: Computer Vision and Pattern Recognition, 2009. CVPR 2009. IEEE Conference on, IEEE (2009) $156-163$

14. Krishnan, D., Tay, T., Fergus, R.: Blind deconvolution using a normalized sparsity measure. In: Computer Vision and Pattern Recognition (CVPR), 2011 IEEE Conference on, IEEE (2011) 233-240 
15. Xu, L., Zheng, S., Jia, J.: Unnatural 10 sparse representation for natural image deblurring. In: Proceedings of the IEEE Conference on Computer Vision and Pattern Recognition. (2013) 1107-1114

16. Mairal, J., Sapiro, G., Elad, M.: Learning multiscale sparse representations for image and video restoration. Multiscale Modeling \& Simulation 7 (2008) 214-241

17. Rubinstein, R., Bruckstein, A.M., Elad, M.: Dictionaries for sparse representation modeling. Proceedings of the IEEE 98 (2010) 1045-1057

18. Elad, M., Yavneh, I.: A plurality of sparse representations is better than the sparsest one alone. IEEE Transactions on Information Theory 55 (2009) 47014714

19. Aharon, M., Elad, M., Bruckstein, A.: K-svd: An algorithm for designing overcomplete dictionaries for sparse representation. IEEE Transactions on Signal Processing 54 (2006) 4311-4322

20. Dong, W., Zhang, L., Shi, G., Li, X.: Nonlocally centralized sparse representation for image restoration. IEEE Transactions on Image Processing 22 (2013) 16201630

21. Daubechies, I., DeVore, R., Fornasier, M., Güntürk, C.S.: Iteratively reweighted least squares minimization for sparse recovery. Communications on Pure and Applied Mathematics 63 (2010) 1-38

22. Zhang, L., Zhang, L., Mou, X., Zhang, D.: Fsim: a feature similarity index for image quality assessment. IEEE transactions on Image Processing 20 (2011) 2378-2386

23. Kheradmand, A., Milanfar, P.: A general framework for regularized, similaritybased image restoration. IEEE Transactions on Image Processing 23 (2014) 51365151

24. Portilla, J.: Image restoration through 10 analysis-based sparse optimization in tight frames. In: 2009 16th IEEE International Conference on Image Processing (ICIP), IEEE (2009) 3909-3912

25. Beck, A., Teboulle, M.: Fast gradient-based algorithms for constrained total variation image denoising and deblurring problems. IEEE Transactions on Image Processing 18 (2009) 2419-2434

26. Beveridge, J.R., Phillips, J., Bolme, D.S., Draper, B., Givens, G.H., Lui, Y.M., Teli, M.N., Zhang, H., Scruggs, W.T., Bowyer, K.W., et al.: The challenge of face recognition from digital point-and-shoot cameras. In: Biometrics: Theory, Applications and Systems (BTAS), 2013 IEEE Sixth International Conference on, IEEE (2013) 1-8

27. Danielyan, A., Katkovnik, V., Egiazarian, K.: Bm3d frames and variational image deblurring. IEEE Transactions on Image Processing 21 (2012) 1715-1728 